\title{
ATENDIMENTOS DE EMERGENCIA POR CAUSAS EXTERNAS NÃO INTENCIONAIS ENVOLVENDO IDOSOS DE FLORIANÓPOLIS SC: VIVA 2006-2007
}

\author{
Flavia Felipe ${ }^{1}$ \\ Pamela Silva Mesadri² \\ Silvia Teixeira ${ }^{3}$ \\ Janaina dos Santos ${ }^{4}$ \\ Andreia Pelegrini ${ }^{5}$
}

resumo

O presente estudo tem como objetivo descrever o perfil dos atendimentos de urgência e emergência por acidentes não intencionais em idosos da capital do estado de Santa Catarina. Participaram do

1 Graduanda em Educação Física. Universidade do Estado de Santa Catarina (UDESC). E-mail: flaviaa.felipe@hotmail.com.

2 Graduada em Educação Física. Universidade do Estado de Santa Catarina (UDESC). E-mail: pamelamesadri@hotmail.com.

3 Graduada em Enfermagem. Prefeitura Municipal de Florianópolis. Secretaria Municipal de Saúde. Diretoria de Vigilância e Saúde. Gerência de Vigilância Epidemiológica. E-mail: silvialineira@hotmail.com.

4 Graduada em Enfermagem. Prefeitura Municipal de Florianópolis. Secretaria Municipal de Saúde. Diretoria de Vigilância e Saúde. Gerência de Vigilância Epidemiológica. E-mail: janainaenf@gmail.com.

5 Graduada em Educação Física. Doutora em Educação Física. Professora Adjunta da Universidade do Estado de Santa Catarina (UDESC), vinculada ao Departamento de Educação Física e ao Programa de Pós-Graduação em Ciências do Movimento Humano. E-mail: andreia.pelegrini@udesc.br. 
estudo idosos acima de 60 anos que foram atendidos nos serviços de urgência e emergência do município de Florianópolis, SC, no período de 2006 ( $n=40)$ e 2007 ( $n=17)$, por meio da análise dos dados secundários do Sistema de Vigilância de Violências e Acidentes (VIVA). Foram coletadas informações referentes ao sexo, à idade, à cor da pele, ao grau de escolaridade, à área de domicílio e aos eventos relacionados às injúrias. Em 2006, as causas externas não intencionais predominaram nas mulheres, em idosos de 60 a 69 anos, de cor de pele branca, com escolaridade de primeira a quarta série do ensino fundamental, residentes na área urbana e cujo meio de transporte mais utilizado foi SAMU/Resgate. Em 2007, as causas externas foram mais evidentes nos idosos de 60 a 69 anos, com ensino fundamental, de cor de pele branca, e domiciliados na área urbana. Utilizaram o veículo particular para chegar ao hospital. Em ambos os períodos, o tipo de ocorrência que prevaleceu foram quedas na residência. A natureza da lesão mais frequente foi a de fratura nos membros inferiores. A maioria dos idosos teve alta e encaminhamento ambulatorial, sem registro de óbito. Considera-se de suma importância o estabelecimento de iniciativas de prevenção voltados para idosos e que integrem as práticas de saúde coletiva e do cuidado individual de idosos.

palavras-chave

Acidentes Domésticos. Causas Externas. Saúde do Idoso. Políticas Públicas.

1 Introdução

O Brasil passa por uma transição demográfica e populacional, em que observa-se um aumento da população idosa (FREIRE et al., 2013). Essa mudança, consequentemente, acarreta gastos elevados para os serviços públicos de saúde, em função do maior desenvolvimento de doenças cardiovasculares e osteomioarticulares durante essa fase da vida (GAWRYSZEWSKI; MELLO, 2000). Além disso, destaca-se a maior incidência de causas externas em idosos devido à maior suscetibilidade em função do processo de envelhecimento (LEITE et al., 2006), o que também gera gastos elevados com assistência médica no Sistema Único de Saúde, que tem por responsabilidade a atenção ao trauma da vítima (MELLO; KOIZUMI, 2004). A partir disso, as causas externas não intencionais são cada vez mais frequentes, o que merece maior atenção dos 
profissionais e serviços da área da saúde (GAWRYSZEWSKI; MELLO, 2000). No Brasil, especificamente, as causas externas correspondem ao maior gasto médio e custo/dia de internação (MELLO; KOIZUMI, 2004).

As causas externas são classificadas como acidentes ou violência, na qual causam injúrias à saúde, podendo ser por causas acidentais como atropelamentos, quedas, envenenamentos, afogamentos, acidentes de trânsito, de trabalho e outros tipos de acidentes ou intencionais relacionados às agressões, às lesões autoprovocadas, aos homicídios e aos suicídios (OMS, 2000). Segundo o Ministério da Saúde (BRASIL, 2010), as causas externas são apontadas como a sétima causa de óbito entre os idosos e a terceira maior causa entre a população em geral.

No Brasil, iniciativas têm sido propostas no sentido de identificar os acidentes por causas externas de pacientes atendidos nos serviços de urgência e emergência, sendo denominado Sistema de Vigilância de Violências e Acidentes (VIVA), o qual tem sido conduzido nas capitais brasileiras e no Distrito Federal, além de algumas cidades brasileiras (BRASIL, 2009). O VIVA, além de obter informações sobre o comportamento destes agravos, busca incentivar a formação de redes de atenção e proteção às pessoas vítimas de acidentes e violências, garantindo assim a promoção da saúde e a cultura de paz (BRASIL, 2009).

Diante de tais informações, faz-se interessante identificar os grupos mais vulneráveis aos acidentes por causas externas, para que sejam adotadas medidas preventivas pelos sistemas de vigilância em saúde. Neste sentido, o presente estudo tem como objetivo descrever o perfil dos atendimentos de urgência e emergência por acidentes não intencionais em idosos da capital do estado de Santa Catarina.

\section{Materiais e método}

O presente estudo, com delineamento transversal, analisa por meio de dados secundários coletados das fichas de notificação do VIVA, referente aos casos de idosos atendidos nos serviços de urgência e emergência de Florianópolis por causas externas não intencionais no período de 2006 e 2007. As notificações ocorreram no período de trinta dias consecutivos entre os meses de setembro e novembro dos respectivos anos. Dentre os serviços de urgência e emergência de Florianópolis, foram considerados elegíveis dois serviços, nomeadamente o Hospital Governador Celso Ramos e o Hospital Infantil Joana de Gusmão (BRASIL, 2009). 
Foram coletadas informações sobre a identificação do paciente (idade, sexo, cor de pele, escolaridade, meio de transporte utilizado para chegar ao hospital, presença ou ausência de deficiência), local de ocorrência (residência, escola, via pública, trabalho, bar, local de prática de esporte), tipo de acidentes (queda, queimaduras, outros), parte do corpo atingida e evolução dos casos (alta, hospitalização, óbito). Visando a padronização da coleta de dados, foram realizados treinamentos pela equipe da Coordenação de Vigilância de Doenças e Agravos Não Transmissíveis da Secretaria de Vigilância em Saúde do Ministério da Saúde (CGDANT/SVS/MS) junto aos técnicos da Secretaria de Saúde do município de Florianópolis (BRASIL, 2010). As entrevistas foram realizadas por acadêmicos de Enfermagem e de Medicina e por profissionais da área da saúde previamente treinados sob supervisão de técnicos da Secretaria de Saúde. A cada paciente admitido no setor de emergência em decorrência das causas externas, os entrevistadores iniciavam a abordagem do paciente ou acompanhante (quando o paciente era menor ou encontrava-se impossibilitado de responder) para solicitar autorização e iniciar a entrevista (BRASIL, 2010).

A assinatura do Termo de Consentimento Livre e Esclarecido foi substituída por consentimento verbal do paciente ou por seu responsável. Conforme o que preconiza a Resolução n.o 466/2012 do Conselho Nacional de Saúde, garantiu-se total anonimato e privacidade aos pacientes, profissionais e gestores dos serviços onde as entrevistas foram realizadas.

Os dados foram tabulados no Setor de Vigilância Epidemiológica e, posteriormente, transferidos para o Ministério da Saúde para consolidação e composição da base de dados nacional. Também foi realizada uma etapa específica para a identificação e exclusão de registros duplicados a partir do uso do programa Link Plus. Considerando-se a semelhança dos resultados observados nos dois anos em que a pesquisa foi realizada, algumas características foram analisadas tomando-se apenas os resultados de 2006. Utilizou-se estatística descritiva (distribuição de frequência) para caracterizar as variáveis investigadas. O teste qui-quadrado foi utilizado para verificar a associação do tipo de ocorrência com a faixa etária (60-69, 70-79 e 80 anos ou mais). Todas as análises foram conduzidas utilizando o pacote estatístico Epi Info for Windows (versão 3.5.1). 
Na pesquisa realizada em 2006, foram registrados 971 atendimentos nos serviços de urgência e emergência do município de Florianópolis, dos quais 40 foram de idosos com 60 anos ou mais. Em 2007, foram registrados 1.154 pacientes, dos quais 17 foram idosos.

As características gerais dos idosos atendidos nos serviços de urgência e emergência referentes aos anos de 2006 e 2007 são apresentadas na Tabela 1. Em 2006, observou-se que as causas externas foram mais frequentes nas mulheres, com idade de 60 a 69 anos, de cor de pele branca, com grau de escolaridade de $1^{\underline{a}}$ a $4^{\underline{a}}$ série do ensino fundamental e domiciliados na área urbana. O meio de transporte mais utilizado para chegar ao hospital foi o SAMU/resgate e a maioria não apresentava nenhum tipo de deficiência física. No ano de 2007, as notificações predominaram no sexo masculino, na faixa etária de 60 a 69 anos, cor da pele branca, com ensino fundamental e residentes na área urbana. $\mathrm{O}$ veículo particular foi o meio de transporte mais utilizado para chegar ao hospital. Ressalta-se que a maioria não apresentou nenhum tipo de deficiência física.

Tabela 1 - Características gerais dos idosos atendidos nos serviços de urgência e emergência de Florianópolis, SC: VIVA 2006-2007.

\begin{tabular}{l|c|c}
\hline \multirow{2}{*}{ Variáveis } & $\mathbf{2 0 0 6}$ & $\mathbf{2 0 0 7}$ \\
\cline { 2 - 3 } & $\mathbf{n}(\mathbf{\%})$ & $\mathbf{n}(\mathbf{\%})$ \\
\hline Masculino & $\mathbf{( n = 4 0 )}$ & $\mathbf{( n = 1 7 )}$ \\
\hline Feminino Faixa etária (anos) & $12(30,0)$ & $9(52,9)$ \\
\hline 60-69 Cor de pele & $28(70,0)$ & $8(47,1)$ \\
\hline $70-79$ & $\mathbf{( n = 4 0 )}$ & $\mathbf{( n = 1 7 )}$ \\
\hline 80 anos ou mais & $17(42,5)$ & $\mathbf{8}(47,1)$ \\
\hline & $14(35,0)$ & $\mathbf{2 ( 1 1 , 8 )}$ \\
\hline Branca & $9(22,5)$ & $\mathbf{( n = 1 7 )}$ \\
\hline Parda & $\mathbf{( n = 4 0 )}$ & $17(100,0)$ \\
\hline
\end{tabular}




\begin{tabular}{|c|c|c|}
\hline Escolaridade & $(n=40)$ & $(n=17)$ \\
\hline Sem escolaridade & $5(12,5)$ & $1(5,9)$ \\
\hline $1^{\mathrm{a}} \mathrm{a} 4^{\mathrm{a}}$ série incompleta do EF & $14(35,0)$ & $3(17,6)$ \\
\hline $4^{\text {a }}$ série completa do EF & $3(7,5)$ & $3(17,6)$ \\
\hline $5^{\mathrm{a}}$ a $8^{\mathrm{a}}$ série incompleta do EF & $5(12,5)$ & $1(5,9)$ \\
\hline Ensino fundamental completo & $2(5,0)$ & $3(17,6)$ \\
\hline Ensino médio completo & $5(12,5)$ & $2(11,8)$ \\
\hline Ignorado & $1(2,5)$ & $3(17,6)$ \\
\hline Área de domicílio & $(n=35)$ & $(n=16)$ \\
\hline Urbana & $33(94,3)$ & $16(100,0)$ \\
\hline Rural & $1(2,9)$ & - \\
\hline Ignorado & $1(2,9)$ & - \\
\hline Como chegou ao hospital & $(n=40)$ & $(n=17)$ \\
\hline A pé & $2(5,0)$ & - \\
\hline Veículo particular & - & $10(58,8)$ \\
\hline SAMU/Resgate* & $26(65,0)$ & $1(5,9)$ \\
\hline Ambulância & $2(5,0)$ & $3(17,6)$ \\
\hline Resgate & $3(15,0)$ & $2(11,8)$ \\
\hline Transporte coletivo & $6(15,0)$ & $1(5,9)$ \\
\hline Outro & $1(2,5)$ & - \\
\hline Deficiência & $(n=39)$ & $(n=17)$ \\
\hline Sim & $15(38,5)$ & $2(11.8)$ \\
\hline Não & $23(59,0)$ & $15(88,2)$ \\
\hline Ignorado & $1(2,6)$ & - \\
\hline
\end{tabular}

* termo utilizado no VIVA 2006

Fonte: Tabela elaborada pelas autoras.

A Tabela 2 descreve as características dos eventos relacionados aos idosos atendidos nos serviços de urgência e emergência de Florianópolis/SC nos anos de 2006 e 2007. Em 2006, o tipo de ocorrência que mais prevaleceu foi quedas e, quanto ao local de ocorrência, predominou a residência. Em relação à atividade praticada no momento do acidente, a maior frequência foi "outros" 
seguido de "trabalhando". Quanto à natureza da lesão, a maioria teve fratura e os membros inferiores foram a parte do corpo mais atingida. Após o primeiro atendimento de emergência, a maioria dos pacientes evoluiu para alta. Em 2007, as quedas foram os tipos de ocorrência que mais predominaram e a residência foi o local de maior ocorrência dos acidentes. Quanto à natureza da lesão, a maioria apresentou corte/perfuração/laceração e os membros superiores foram a parte do corpo mais atingida. A evolução do caso foi considerada alta.

Tabela 2 - Características dos eventos relacionados aos idosos atendidos nos serviços de urgência e emergência de Florianópolis, SC: VIVA 2006-2007.

\begin{tabular}{|c|c|c|}
\hline Variáveis & $\begin{array}{l}2006 \\
\text { n (\%) }\end{array}$ & $\begin{array}{l}2007 \\
\text { n (\%) }\end{array}$ \\
\hline Tipo de ocorrência & $(n=40)$ & $(n=17)$ \\
\hline Queda & $35(87,5)$ & $14(82,4)$ \\
\hline Outros acidentes & $5(12,5)$ & $3(17,6)$ \\
\hline Local de ocorrência & $(n=40)$ & $(n=17)$ \\
\hline Residência & $30(75,0)$ & $8(47,1)$ \\
\hline Via pública (rua) & $5(12,5)$ & $5(29,4)$ \\
\hline Via pública (rodovia) & $1(2,5)$ & - \\
\hline Trabalho & $1(2,5)$ & - \\
\hline Bar ou similar & $2(5,0)$ & - \\
\hline Comércio/serviços & - & $1(5,9)$ \\
\hline Outro & $1(2,5)$ & $3(17,6)$ \\
\hline Atividade realizada & $(n=40)$ & \\
\hline Trabalhando & $15(37,5)$ & - \\
\hline No trajeto para o trabalho & - & - \\
\hline Estudando & - & - \\
\hline Praticando esporte & - & - \\
\hline Viajando & - & - \\
\hline Recreação/lazer & $7(17,5)$ & - \\
\hline Outro & $17(42,5)$ & - \\
\hline lgnorado & $1(2,5)$ & - \\
\hline
\end{tabular}




\begin{tabular}{|c|c|c|}
\hline Natureza da lesão & $(n=40)$ & $(n=17)$ \\
\hline Fratura & $13(32,5)$ & $3(17,6)$ \\
\hline Entorse/luxação & $7(17,5)$ & $2(11,8)$ \\
\hline Corte/perfuração/laceração & $6(15,0)$ & $6(35,3)$ \\
\hline Contusão & $10(25,0)$ & $5(29,4)$ \\
\hline Outros & $1(2,5)$ & $1(5,9)$ \\
\hline Ignorado & $3(7,5)$ & - \\
\hline Parte do corpo atingida & $(n=40)$ & $(n=17)$ \\
\hline Cabeça e face & - & $3(17,6)$ \\
\hline Pescoço & - & $1(5,9)$ \\
\hline MMII & $12(30,0)$ & $4(23,5)$ \\
\hline MMSS & $11(27,5)$ & $7(41,2)$ \\
\hline Tórax & $1(2,5)$ & - \\
\hline Tórax/dorso & - & $1(5,9)$ \\
\hline Coluna e medula & - & $1(5,9)$ \\
\hline Coluna & $1(2,5)$ & - \\
\hline MMSS/MMII & $1(2,5)$ & - \\
\hline Cabeça & $5(12,5)$ & - \\
\hline Abdome & $1(2,5)$ & - \\
\hline Cabeça e Boca & $1(2,5)$ & - \\
\hline Cabeça/MMSS & $3(7,5)$ & - \\
\hline Torax/MMII & $2(5,0)$ & - \\
\hline MMSS/MMII/Cabeça & $1(2,5)$ & - \\
\hline Coluna/Tórax & $1(2,5)$ & - \\
\hline Evolução do caso & & $(n=17)$ \\
\hline Alta & $26(65,0)$ & $8(47,1)$ \\
\hline Encaminhamento ambulatorial & $1(2,5)$ & $3(17,6)$ \\
\hline Encaminhamento hospitalar & $10(25,0)$ & - \\
\hline Internação hospitalar & - & $5(29,4)$ \\
\hline Evasão/Fuga & $1(2,5)$ & - \\
\hline lgnorado & $2(5,0)$ & $1(5,9)$ \\
\hline
\end{tabular}

- a variável atividade realizada não foi coletada no Inquérito VIVA-2007.

Fonte: Tabela elaborada pelas autoras. 
A partir dos resultados da Tabela 3, é possível observar que não houve associação entre o tipo de acidente e o grupo etário quando estratificados por ano (2006 e 2007). Em 2006, observa-se que ocorreu uma redução dos acidentes por quedas conforme o avanço da faixa etária, enquanto o número de ocorrências por "outros acidentes" diminuiu com o aumento da idade. Em 2007, houve diminuição dos casos de quedas conforme o avanço da faixa etária e aumento dos "outros acidentes" entre as faixas etárias de 60-69 e 70-79, seguida de redução na faixa etária de 80 anos ou mais.

Tabela 3 - Tipos de acidentes atendidos nos serviços de urgência e emergência de Florianópolis, SC, estratificados por faixa etária. VIVA 2006-2007.

\begin{tabular}{c|c|c|c|c|c}
\hline \multicolumn{2}{c|}{ Variáveis } & \multicolumn{4}{c}{ Grupo etário (idade) } \\
\hline \multirow{2}{*}{ Ano } & Tipo de acidente & $\mathbf{6 0 - 6 9}$ & $\mathbf{7 0 - 7 9}$ & $\mathbf{8 0 ~ o u +}$ & p-valor \\
\cline { 2 - 6 } & Queda & $13(76,5)$ & $13(92,9)$ & $\mathbf{9 ( 1 0 0 , 0 )}$ & \\
\cline { 2 - 6 } 2007 & Outros acidentes & $4(23,5)$ & $1(7,1)$ & - & 0,170 \\
\cline { 2 - 6 } & Queda & $7(87,5)$ & $5(71,4)$ & $2(100,0)$ & 0.563 \\
\hline
\end{tabular}

Fonte: Tabela elaborada pelas autoras.

\section{Discussão}

Os resultados referentes aos inquéritos do VIVA de 2006 e 2007 do município de Florianópolis, SC, revelaram semelhança de ocorrência entre homens e mulheres. Ademais, observaram-se maiores ocorrências nos indivíduos de 60-69 anos, da cor de pele branca e residentes na área urbana. O tipo de ocorrência que mais prevaleceu foram quedas, o local de ocorrência que mais predominou foi a residência, os pacientes apresentaram fratura e os membros inferiores e superiores foram as partes do corpo mais atingidas.

O número de notificações por causas externas foi semelhante entre homens e mulheres nos anos de 2006 e 2007. Estes dados divergem dos achados por Grden et al. (2014), os quais identificaram predominância dos acidentes por causas externas no sexo feminino, o que pode estar atrelado às atividades domésticas realizadas pelas mulheres, geralmente aposentadas ou donas de casa, e também pelas barreiras arquitetônicas do ambiente doméstico.

Quanto à faixa etária, as causas externas foram mais predominantes nos indivíduos de 60-69 anos. Dados da literatura apontam um acréscimo 
substancial de acidentes por causas externas conforme o aumento da idade (SECULI et al., 2004). Esse fato pode estar atrelado à perda de equilíbrio e a alterações na massa muscular e óssea (SECULI et al., 2004). Ademais, destaca-se a observância cada vez maior das pessoas dessa faixa etária apresentarem autonomia e independência, pois os idosos realizam atividades sociais e de lazer e estão ainda inseridas no mercado de trabalho (GRDEN et al., 2014). Além disso, com o aumento da expectativa de vida e as iniciativas de políticas públicas visando o envelhecimento ativo, tem-se observado um aumento na parcela de idosos com melhores condições de vida e que contribuem ativamente dentro da comunidade (OLIVEIRA et al., 2014). Essa mudança pode gerar o aumento do número de acidentes em ambientes externos. A maioria das notificações ocorreu nos idosos com grau de escolaridade inferior a oito anos de estudo, o que de fato pode estar relacionado à alfabetização nas décadas de 1920-1940, quando as pessoas não eram incentivadas ao estudo nem exigidas a frequentar o ambiente escolar (FREIRE et al., 2013).

O meio de locomoção mais utilizado para a chegada ao hospital foi o Resgate/SAMU, que, conforme Minayo e Deslandes (2009), representa um melhor atendimento para a população em situações de acidentes, pois a locomoção por meio deste está relacionado à maior segurança. Apesar disso, o serviço ainda necessita de melhorias como a inserção de ambulâncias para agilizar o atendimento. Além desses dados, a residência foi o local de maior ocorrência das causas externas. Estes achados corroboram aos de Grden et al. (2014), os quais verificaram que dos 324 atendimentos, mais da metade decorrem de eventos no próprio domicílio (52,8\%). O ambiente físico em que o idoso se encontra está diretamente associado ao número de acidentes por causas externas, principalmente aqueles atrelados à maior ocorrência de quedas, em virtude de tapetes soltos, piso escorregadio, objetos em áreas de circulação, ausência de barras de apoio e corrimões, móveis instáveis e iluminação inadequada (AMBROSE; PAUL; HAUSDORFF, 2013).

O tipo de ocorrência com maior predominância foram as quedas nos dois períodos investigados. Estes dados são preocupantes, tendo em vista que as quedas apresentam diversos impactos na vida do idoso, e sua ocorrência está relacionada às altas taxas de morbi-mortalidade, deterioração funcional, hospitalização e o consumo de serviços de saúde, além das consequências diretamente ligadas a quedas, nas quais os idosos restringem suas atividades devido a dores, incapacidades e ao medo de cair (FABRÍCIO; RODRIGUES; 
COSTA JUNIOR, 2004). Um estudo realizado no Brasil aponta que, no ano de 2000, foram registrados mais de 2 mil mortes ocasionadas por quedas, sendo elas ocorridas em idosos com 60 anos ou mais (GAWRYSZEWSKI; JORGE; KOIZUMI, 2004). Este fato associado ao local de ocorrência (residência) pode estar relacionado à disposição dos móveis e objetos em locais inadequados, o que facilitaria o acontecimento das quedas. Consequentemente, torna-se de suma importância o cuidado com a disposição dos objetos nas residências, o que contribui para a prevenção de ocorrências de quedas (ANDRADE et al., 2012). Além dessa recomendação, o estudo de Siqueira et al. (2007), realizado no Rio Grande do Sul em idosos, associou a prevalência de quedas com a idade avançada, o sedentarismo e a autopercepção de saúde como sendo ruim e maior número de medicações referidas para o uso contínuo.

No ano de 2006, observou-se que, quanto à natureza da lesão, as fraturas ocorreram em maior número nos membros inferiores. Segundo Lustosa e Bastos (2009), quando a fratura é ocasionada nas áreas proximais do fêmur em idosos, elas representam um sério problema na saúde pública, pois além de obter-se nesses casos uma alta taxa de morbidade e mortalidade, esse tipo de tratamento tem elevados custos econômicos. Essa ocorrência de lesão dá-se com maior frequência em mulheres devido a um pior estado funcional, a uma maior morbidade, a uma maior exposição às atividades domésticas e à osteoporose (FABRÍCIO; RODRIGUES; COSTA JUNIOR, 2004).

Este estudo apresentou algumas limitações, dentre as quais destacam-se as limitações relacionadas ao preenchimento do questionário, pois, de acordo com o estado emocional em que o paciente chegava aos serviços de urgência e emergência, o indivíduo não apresentava condições emocionais e/ou físicas para responder e,em alguns momentos, esse procedimento foi realizado pela equipe médica. Os dados encontrados no presente estudo não podem ser expandidos para toda a população de Florianópolis, tendo em vista o método utilizado para a coleta de dados durante o período de 30 dias consecutivos. Além disso, a diferença das questões no instrumento de coleta nos anos de 2006 e 2007 também dificultou a comparação entre as variáveis. Ademais, destaca-se que a principal vantagem do presente estudo relaciona-se a possibilidade de uma investigação por inquérito com o intuito de analisar os acidentes mais frequentes e os grupos mais vulneráveis notificados nos serviços de urgência e de emergência de referência do município. 
Conclui-se que os acidentes por causas externas ocorreram com maior frequência no sexo feminino, na faixa etária de 60 a 69 anos, sendo a queda o tipo de ocorrência mais frequente e a residência o local, de maior ocorrência. Com os dados obtidos, é possível ter maior conhecimento sobre o perfil epidemiológico dos idosos, o que possibilita o planejamento de ações específicas, na assistência à saúde básica, tais como a elaboração de métodos preventivos e intervenções terapêuticas que sejam direcionadas para manter ou melhorar a capacidade funcional e prevenir os danos físicos e internações hospitalares em idosos, reduzindo os custos que as quedas geram para o sistema de saúde.

\author{
EMERGENCY CALLS FOR UNINTENTIONAL \\ EXTERNAL CAUSES INVOLVING SENIORS \\ FROM FLORIANÓPOLIS, SC: VIVA 2006-2007
}

abstract

The aim of this study was to describe the profile of urgent and emergency care and unintentional injuries involving seniors of the state capital of Santa Catarina. Study participants were aged 60 years old who were treated in emergency from Florianópolis, SC, from $2006(n=40)$ and $2007(n=17)$, through the analysis of secondary data collected from reporting Surveillance System for Violence and Accidents (VIVA). Information on gender, age, skin color, level of education and area of residence and from the events related to injury were collected. In 2006, unintentional external causes predominated in 60-69-year-old women, white skin color, schooling from first to fourth grade elementary incomplete, teaching residents in the urban area and the means of transport most used was SAMU/ Rescue. In 2007, external causes were more evident in 60-69-year-olds with primary school, white skin color, resedent in urban area. The private vehicle was the most used to go to the hospital. In both periods, the occurrence of which prevailed was falls in residence. The nature of the most frequent injury was fracture of the lower limbs. Most seniors were discharged and outpatient referral without death record. It is considered of paramount importance to establish prevention initiatives aimed at elderly and integrate the practices of individual and collective health care of the elderly. 
Domestic Accidents. External Causes. Health of the Elderly. Public Policy.

referências

AMBROSE, Anne Felicia; PAUL, Geet; HAUSDORFF, Jeffrey M. Risk factors for falls among older adults: a review of the literature. Maturitas, Oxford, v. 75, n. 1, p. 51-61, May 2013.

ANDRADE, Silvânia Suely Caribé de Araújo et al. Perfil das vítimas de violências e acidentes atendidas em serviços de urgência e emergência selecionados em capitais brasileiras: Vigilância de Violências e Acidentes, 2009. Epidemiologia e Serviços de Saúde, Brasília, v. 21, n. 1, p. 21-30, jan./mar. 2012.

BRASIL. Ministério da Saúde. Secretaria de Vigilância em Saúde. Departamento de Análise de Situação de Saúde. Viva: vigilância de violências e acidentes, 2006 e 2007. Brasília: Ministério da Saúde, 2009. Disponível em: < http://bvsms.saude.gov.br/bvs/ publicacoes/viva_vigilancia_violencias_acidentes.pdf> Acesso em: 4 mar. 2016.

Ministério da Saúde. Secretaria de Vigilância em Saúde. Departamento de Análise de Situação de Saúde. Viva: vigilância de violências e acidentes, 2008 e 2009. Brasilia: Ministério da Saúde, 2010. Disponível em: < http://bvsms.saude.gov.br/bvs/ publicacoes/viva_2008_2009_violencias_acidentes.pdf> Acesso em: 4 mar. 2016.

FABRíCIO, Suzele Cristina Coelho; RODRIGUES, Rosalina A. Partezani; COSTA JUNIOR, Moacyr Lobo da. Causas e conseqüências de quedas de idosos atendidos em hospital público. Revista de Saúde Pública, São Paulo, v. 38, n. 1, p. 93-99, fev. 2004.

FREIRE, Glauciane Aparecida et al. Mortalidade Por Causas Externas em Idosos no Paraná, Brasil de 2001-2010. UNOPAR Científica: Ciências Biológicas e da Saúde, Londrina, v. 15, n. 2, p. 161-67, 2013.

GAWRYSZEWSKI, Vilma Pinheiro; JORGE, Maria Helena Prado de Mello. Mortalidade violenta no Município de São Paulo nos últimos 40 anos. Revista Brasileira de Epidemiologia, São Paulo, v. 3, n. 1-3, p. 50-69, abr./dez. 2000.

GAWRYSZEWSKI, Vilma Pinheiro; JORGE, Maria Helena Prado de Mello; KOIZUMI, Maria Sumie. Mortes e internações por causas externas entre idosos no Brasil: o desafio de integrar a saúde coletiva e atenção individual. Revista da Associação Médica Brasileira, São Paulo, v. 50, n. 1, p. 97-103, 2004.

GRDEN, Clóris Regina Blanski et al. Caracterização de idosos vítimas de acidentes por causas externas. Cogitare Enfermagem, Curitiba, v. 19, n. 3, p. 506-513, jul./set. 2014.

LEITE, Marinês Tambara et al. Ocorrência e prevenção de acidentes envolvendo idosos no espaço doméstico. Revista Contexto \& Saúde, ljuí, v. 6, n. 11, p. 83-90, jul./dez. 2006.

LUSTOSA, Lygia Paccini; BASTOS, Eduardo Onofre. Fraturas proximais do fêmur em idosos: qual o melhor tratamento? Acta Ortopédica Brasileira, São Paulo, v. 17, n. 5, p. 309-12, 2009.

MINAYO, Maria Cecília de Souza; DESLANDES, Suely Ferreira. Análise da implantação da rede de atenção às vítimas de acidentes e violências segundo diretrizes da Política Nacional de Redução da Morbimortalidade sobre Violência e Saúde. Ciência \& Saúde Coletiva, Rio de Janeiro, v. 14, n. 5, p. 1641-1649, nov./dez. 2009. 
OLIVEIRA, Adriana Sarmento et al. Fatores ambientais e risco de quedas em idosos: revisão sistemática. Revista Brasileira de Geriatria e Gerontologia, Rio de Janeiro, v. 17, n. 3, p. 637-645, jul./set. 2014.

ORGANIZAÇÃO MUNDIAL DA SAÚDE. Classificação estatística internacional de doenças e problemas relacionados à saúde. 10. rev. São Paulo: Centro Colaborador da OMS para classificação de Doenças em Português/EDUSP, 1993. v. 1.

SÉCULI SÁNCHEZ, Elisa et al. Las caídas en los mayores de 65 años: conocer para actuar. Atención Primaria, Cataluña, v. 34, n. 4, p. 186-191, sept. 2004.

SIQUEIRA, Fernando Vinholes et al. Prevalência de quedas em idosos e fatores associados. Revista de Saúde Pública, São Paulo, v. 41, n. 5, p. 749-56, out. 2007.

Recebido: 16/09/2014

Aceite Final: 18/04/2016 